\title{
An audit of ingested and aspirated foreign bodies in children at a university hospital in South Africa: The Pietermaritzburg experience
}

\author{
N F Majola, ${ }^{1}$ MB ChB; V Y Kong, ${ }^{1}$ MSc, PhD, MRCS; H Mangray, ${ }^{1}$ FCS (SA); V Govindasamy, ${ }^{1}$ FCS (SA); G L Laing, ${ }^{1}$ MSc, PhD, FCS (SA); \\ D L Clarke, ${ }^{1,2,3}$ MPhil, MBA, PhD, FCS (SA) \\ ${ }^{1}$ Department of Paediatric Surgery, Grey's Hospital, Pietermaritzburg, South Africa \\ ${ }^{2}$ Department of Surgery, Nelson R Mandela School of Medicine, College of Health Sciences, University of KwaZulu-Natal, Durban, South Africa \\ ${ }^{3}$ Department of Surgery, Faculty of Health Sciences, University of the Witwatersrand, Johannesburg, South Africa
}

Corresponding author: V Y Kong (victorywkong@yahoo.com)

\begin{abstract}
Background. The ingestion or aspiration of foreign bodies (FBs) by children is a common problem around the world. Our centre in Pietermaritzburg, South Africa, has a dedicated paediatric surgical service, and all patients with an ingested or aspirated FB are managed under the direct care of a paediatric surgeon.

Objectives. To review our centre's experience with this problem by means of a retrospective audit and use the data to develop and refine appropriate local management guidelines.

Methods. Grey's Hospital has a hybrid electronic medical registry (HEMR) that captures patient data on admission, after a procedure and on discharge. The HEMR was reviewed and all patients with an appropriate International Statistical Classification of Diseases and Related Health Problems, 10th revision (ICD-10) code indicating an ingested or aspirated FB were identified and retrieved for review.

Results. A total of 105 cases of FB ingestion or aspiration in children $<12$ years of age from January 2012 to December 2014 were identified from the HEMR. The patients' ages ranged from 4 months to 10 years (mean 3 years and 6 months), and $59.0 \%$ ( $n=62)$ were male and $41.0 \%(n=43)$ female. A total of 107 FBs were removed (two patients each had two coins removed). The commonest FBs were coins ( $n=77,71.9 \%)$, followed by batteries $(n=6,5.6 \%)$, plastic toys $(n=5,4.7 \%)$, buttons $(n=5,4.7 \%)$, screws/washers $(n=3,2.8 \%)$, seeds $(n=2,1.9 \%)$, needles $(n=2,1.9 \%)$, bones $(n=2,1.9 \%)$, a marble $(n=1,0.9 \%)$, a rubber eraser $(n=1,0.9 \%)$, a curtain hook $(n=1,0.9 \%)$, a nail $(n=1,0.9 \%)$ and a wood speck $(n=1,0.9 \%)$. Of the FBs, $67(62.6 \%)$ were in the oesophagus, $17(15.9 \%)$ in the respiratory system, $14(13 \%)$ in the intestine and $9(8.4 \%)$ in the oral cavity. The average time from ingestion/aspiration to presentation was $<48$ hours. Of the FBs, 67 (62.6\%) were removed via rigid oesophagoscopy and $13(12.1 \%)$ via rigid bronchoscopy, $13(12.1 \%)$ were passed rectally, and $9(8.4 \%)$ were removed via grasping forceps in the oral cavity, 4 (3.7\%) via thoracotomy and $1(0.9 \%)$ via emergency laparotomy. A total of 15 complications included mucosal ulceration/slough $(n=6,40.0 \%)$, oesophageal perforation $(n=3,20.0 \%)$, aspiration pneumonia $(n=3,20.0 \%)$, and tracheal perforation, lung collapse and contact bleed $(n=1$ each, 6.7\%). No patient presented in respiratory distress or needed emergency airway management, and there were no deaths.

Conclusions. The development of a dedicated paediatric surgery service and the implementation of management protocols have resulted in excellent outcomes for this problem.
\end{abstract}

S Afr Med J 2018;108(3):205-209. DOI:10.7196/SAMJ.2018.v108i3.12590

The ingestion or aspiration of foreign bodies (FBs) by children aged $<12$ years is a common problem around the world. ${ }^{[1-6]}$ It can be lifethreatening, and appropriate management of the airway and safe and appropriate retrieval of the $\mathrm{FB}$ are essential. ${ }^{[1,2]}$

\section{Aspirated FBs}

Aspiration of an FB can be life-threatening, as the FB can acutely occlude the proximal airway. Once the FB passes distal to the carina, its location will depend on the age of the patient and the mechanism of aspiration. In children the angle created by the bifurcation of the bronchi is equal in size, and solid FBs have an equal propensity to move into the right or left main bronchus. Aspirated FBs must be removed, as they are associated with a significant risk of complications, but uncontrolled attempts to remove an aspirated FB may result in its further displacement. Organic material may swell and exacerbate inflammation, leading to erosion of the bronchial wall and egress of infected material into the mediastinum. In the airway distal to the obstruction, air trapping may cause emphysema, atelectasis and vasoconstriction.
The priority in these patients is to ensure a patent airway and adequate oxygenation, and clinical examination must identify signs of airway obstruction. A chest radiograph will identify radio-opaque objects, but most aspirated objects are radiolucent and are invisible on a plain chest radiograph. A computed tomography (CT) scan of the chest is the investigation of choice to identify aspirated FBs. A CT scan of the chest shows and defines the anatomy of the lungs and respiratory tract, making it easy to visualise an object lodged in the airway.

Rigid bronchoscopy under general anaesthesia is the generally recommended technique to remove aspirated FBs. The patient can be ventilated during the procedure, and appropriate instruments can be passed through the operating port to remove solid FBs and suction out semi-solid and liquid debris.

\section{Ingested FBs}

Children most commonly swallow smooth, round objects such as coins $^{[1-4]}$ Ingested FBs tend to lodge at natural narrowings of the intestinal tract, the most common sites being the oesophagus at 
the thoracic inlet, the aortic arch and the oesophagogastric (OG) junction. There is a high likelihood that FBs will traverse the intestinal tract spontaneously.

Objects proximal to the OG junction in the oesophagus should be removed, whereas those distal to this landmark can usually be managed conservatively by observation, serial clinical examination and follow-up radiological imaging. ${ }^{[1-6]}$ Complications of ingested FBs are rare, but are well described and usually related to pressure necrosis of the oesophagus, causing perforation and subsequent mediastinal sepsis. Certain characteristics of the ingested FB increase the likelihood of a complication. Sharp objects may lacerate the intestinal tract, large objects such as marbles may become impacted, disc batteries are corrosive, and magnets may trap the luminal wall between them.

\section{Objective}

To review our centre's experience with this problem in order to benchmark our experience with that reported in the national and international literature and use the data to develop and refine appropriate local guidelines for management of this problem.

\section{Methods}

Grey's Hospital in Pietermaritzburg, South Africa (SA), has a hybrid electronic medical registry (HEMR), which captures patient data on admission, after a procedure and on discharge. All data on the HEMR are coded according to the International Statistical Classification of Diseases and Related Health Problems, 10th revision (ICD-10). The registry has ethical approval through the Biomedical Research Ethics Committee of the University of KwaZulu-Natal (ref. no. BCA 221/2013). The HEMR was reviewed and all cases with an appropriate ICD-10 code were identified and retrieved for review. The respiratory tract was defined as extending from the trachea to the bronchioles of the lungs, and the digestive tract as extending from the oesophagus to the rectum. Demographic data, clinical presentation, radiological findings and endoscopic findings were documented, and all the medical records of the 105 cases with full documentation were analysed.

\section{Setting}

Grey's Hospital is a tertiary hospital in the city of Pietermaritzburg in KwaZuluNatal (KZN) Province. It is designated as the tertiary referral hospital for western KZN. The city has a million inhabitants, and the western part of KZN is a rural area with about three million inhabitants. The area is served by three regional hospitals and 19 district hospitals. Grey's Hospital pioneered the development of a dedicated paediatric surgery service in the region. This service produced standardised management algorithms for the management of paediatric FBs. The service is specialist-led, and a consultant paediatric surgeon is present for all FB retrievals.

\section{Management algorithms}

Fig. 1 demonstrates our current management approach to patients with an ingested or aspirated FB. The primary distinction to make is whether the FB has been aspirated into the respiratory tract or ingested into the gastrointestinal tract, or is lodged in the common aero-digestive (oral) tract. The degree of urgency in managing FBs depends very much on whether the object is in the digestive tract or in the airway.

\section{Results}

A total of 105 cases of $\mathrm{FB}$ ingestion or aspiration in children aged $<12$ years from January 2012 to December 2014 were identified from the HEMR. The patients' ages ranged from 4 months to 10 years (mean 3 years and 6 months), and 59.0\% ( $n=62)$ were male and $41.0 \%(n=43)$ female. A total of $107 \mathrm{FBs}$ were removed, two patients each having two coins removed. Of the FBs, 67 $(62.6 \%)$ were found in the oesophagus, 17 $(15.9 \%)$ in the respiratory system, 14 (13\%) in the intestinal tract and $9(8.4 \%)$ in the oral cavity. The commonest FBs removed were coins $(n=77,71.9 \%)$, followed by batteries ( $n=6,5.6 \%)$, plastic toys $(n=5,4.7 \%)$, buttons $(n=5,4.7 \%)$, screws/washers $(n=3,2.8 \%)$, seeds $(n=2,1.9 \%)$, needles $(n=2,1.9 \%)$, bones $(n=2,1.9 \%)$, a marble ( $n=1,0.9 \%)$, a rubber eraser $(n=1,0.9 \%)$, a curtain hook $(n=1,0.9 \%)$, a nail $(n=1,0.9 \%)$ and a wood speck $(n=1,0.9 \%)$. It is important to note that $82.1 \%$ of the FBs in the gastrointestinal tract were coins, whereas in the oesophagus there was an almost equal distribution of the different type of FBs, with only $20.9 \%$ being coins. Figs 2 - 8 show some of the FBs removed or spontaneously passed during the study period. The average time from ingestion/aspiration to presentation was $<48$ hours. Of the FBs, 67 (62.6\%) were removed via rigid oesophagoscopy and 13 (12.1\%) via rigid bronchoscopy, 13 (12.1\%) were passed rectally, and 9 (8.4\%) were removed with grasping forceps in the oral cavity, 4 (3.7\%) via thoracotomy and 1 (0.9\%) via emergency laparotomy. Thoracotomies were performed for oesophageal perforation $(n=1)$, tracheal perforation $(n=1)$, failed removal via bronchoscopy of an FB that was stuck in a bronchus $(n=1)$, and removal of an FB that had progressed too far to be reached via bronchoscopy, as it was in a bronchiole $(n=1)$. Patients who required a second procedure such as thoracotomy to facilitate removal of an $\mathrm{FB}$ or manage complications presented long after ingestion or aspiration, with presentation being 1 week to 6 months later. Emergency laparotomy was performed for removal of a battery in the small bowel to prevent bowel perforation from erosion by battery liquid. A total of 15 complications recorded included mucosal ulceration/slough $(n=6$, $40.0 \%)$, oesophageal perforation $(n=3$, $20.0 \%)$, aspiration pneumonia $(n=3,20.0 \%)$, and tracheal perforation, lung collapse and contact bleed ( $n=1$ each, $6.7 \%$ ). Fortunately no patient presented in respiratory distress

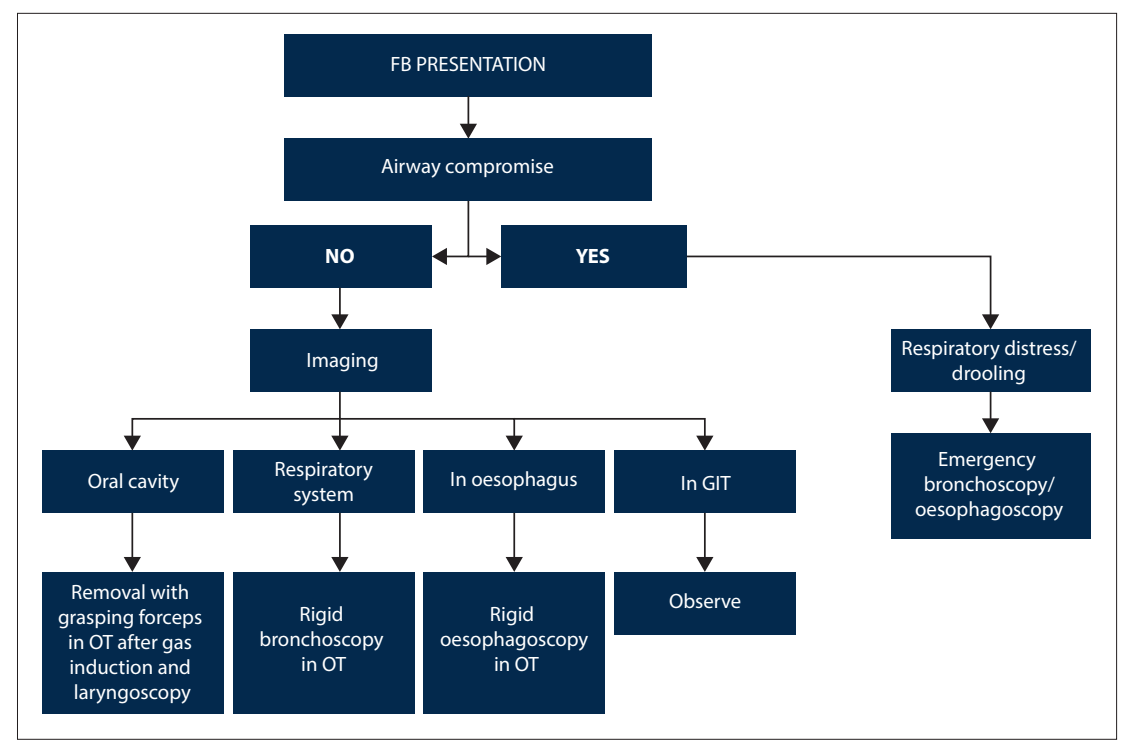

Fig. 1. The management algorithm for FB management in Pietermaritzburg, South Africa. (FB = foreign body; OT = operating theatre; GIT = gastrointestinal tract.) 


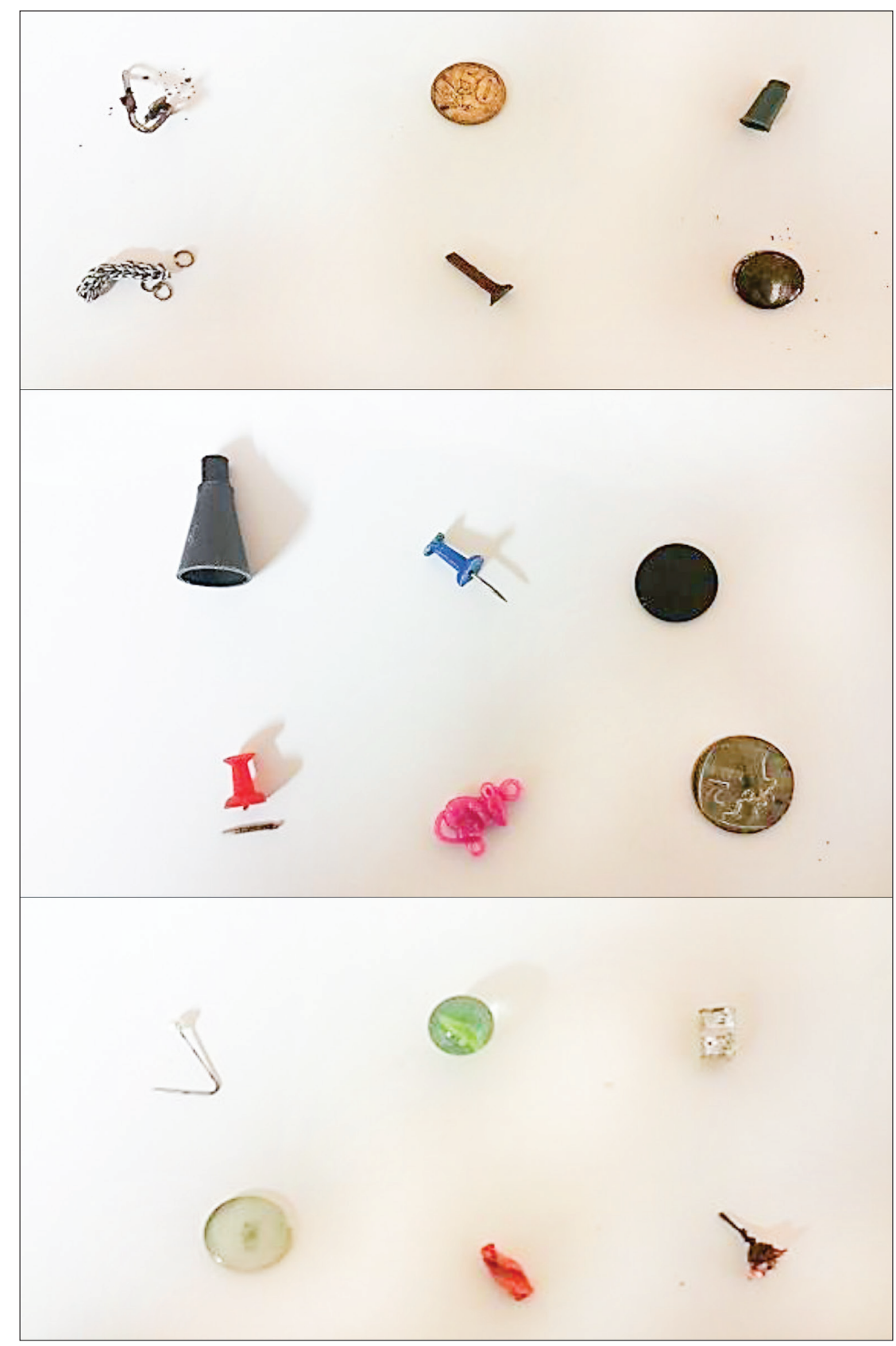

Fig. 2. Some of the foreign bodies removed during the study period.

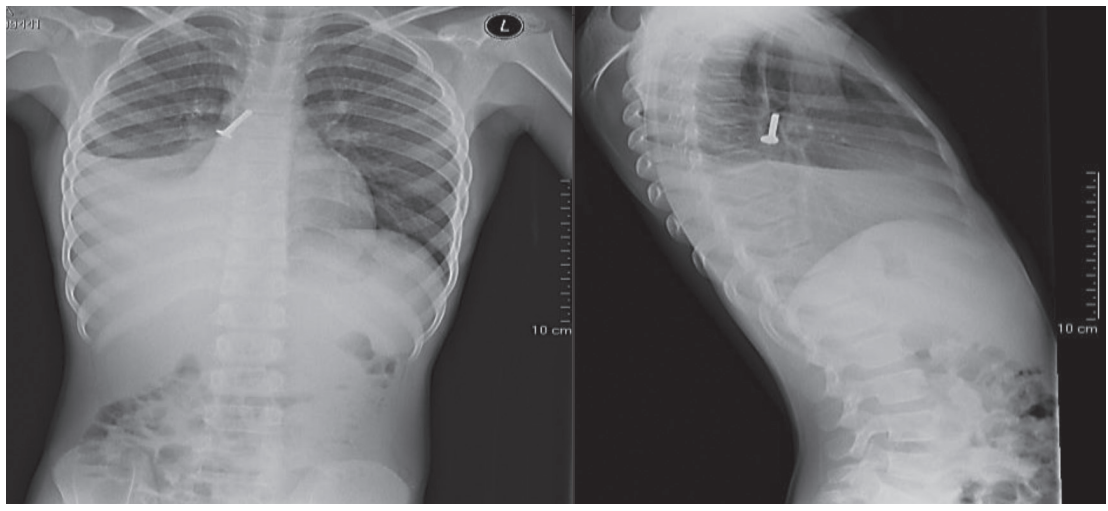

Fig. 3. Screw in the right main bronchus, removed via rigid bronchoscopy, associated with right lower lobe collapse. or needed emergency airway management. There were no deaths.

\section{Discussion}

FB ingestion and aspiration is a common paediatric emergency, and treatment needs to follow well-established principles. ${ }^{[1-8]}$ The primary concern is adequacy of the airway. ${ }^{[1-4]}$ FBs most likely to affect breathing are those in the common aerodigestive tract and those in the proximal airway. ${ }^{[1,2]}$ Emergency airway skills may be needed to deal with these problems, e.g. rigid laryngoscopy and removal of the FB with Magill forceps or needle cricothyroidotomy to temporarily relieve airway obstruction while the patient is in casualty. Once the airway is secured, the next step is to ascertain whether the FB is in the digestive tract or the respiratory tract. This is done by clinical examination and appropriate imaging.

Our series is similar to those reported from other SA institutions. Among the most extensive SA experience has been at Red Cross Children's War Memorial Hospital in Cape Town. ${ }^{[2-4]}$ The authors reported from a dedicated paediatric hospital on 241 patients with ingested $\mathrm{FBs}^{[2]}$ The demographic profile of the patients was similar to ours, and both sexes were equally affected. The ages of the patients ranged from $<1$ year to 12 years, which is similar to our series, with a peak incidence at 3 years. The nature of the ingested objects was also similar to our experience, and the most commonly ingested objects were coins $(28 \%)$, balls $(20 \%)$ and bones $(8 \%)$. The anatomical distribution was also similar, with the most common anatomical site of impaction being the nose (41\%), followed by the oesophagus (20\%), the stomach (14\%) and the bowel (11\%). ${ }^{[2]}$ The Red Cross group removed 57\% of ingested FBs using rigid oesophagoscopy, $19 \%$ were left in situ, 14\% spontaneously dislodged and only $1 \%$ were removed by Foley catheter manipulation. ${ }^{[2,3]}$ We generally do not use the Foley catheter technique, opting for the more controlled environment in the operating theatre.

The management of aspirated FBs remains more challenging than that of ingested FBs, and this is shown by our data. The potential of a threatened airway always exists, and the team managing these patients must include a skilled anaesthesiologist. An FB impacted at the epiglottis can be particularly difficult to manage, as it may be impossible to intubate the patient in this situation. ${ }^{[5-10]}$ Surgical airways are generally contraindicated in small children, and needle cricothyroidotomy may be necessary in select patients to allow 


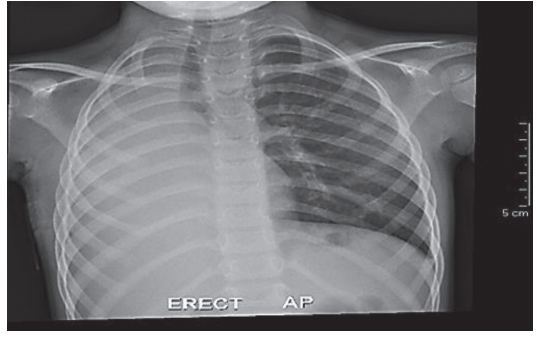

Fig. 4. Collapsed right lung due to a seed in the right main bronchus (the foreign body is not visible on this film). The patient underwent thoracotomy for removal of the seed. temporary jet ventilation. ${ }^{[6-10]}$ Paradoxically, once the patient is relaxed it is generally easy to retrieve such FBs from the back of the throat using Magill forceps or other grasping forceps ${ }^{[1-5]}$ FBs below the epiglottis should be removed with a rigid bronchoscope, which allows for ongoing ventilation of the patient and for the passage of retrieval instruments.

FB aspiration or ingestion is associated with a significant risk of morbidity, as shown by our data. Impacted FBs in the oesophagus can erode through its wall causing mediastinitis, as in one of our cases.

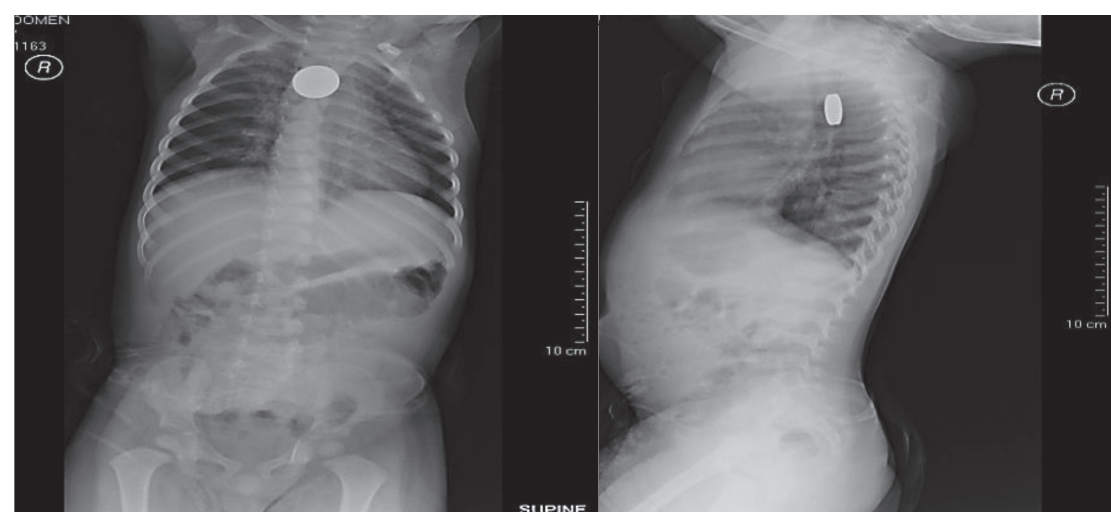

Fig. 5. Coin in the oesophagus, removed via rigid oesophagoscopy.

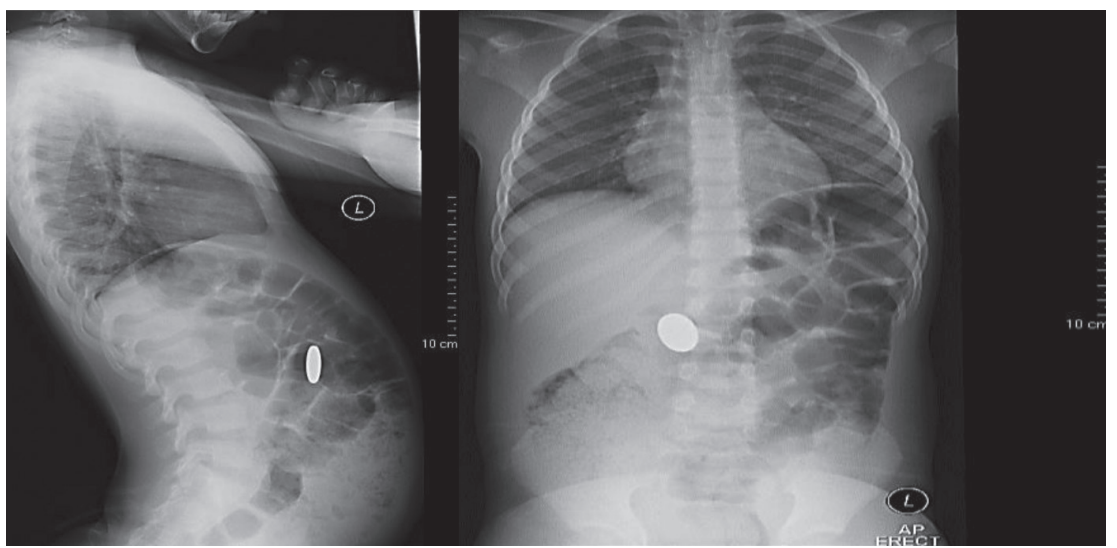

Fig. 6. This coin in the small bowel was conservatively treated and was passed rectally.

Rigid bronchoscopy may be associated with tracheal perforation, especially if the FB is impacted and force is used to dislodge it. We resorted to thoracotomy in four patients. The rate of thoracotomy for aspirated FBs ranges from $1 \%$ to $4 \%{ }^{[1-10]}$ It was high in our series, almost certainly owing to the long delays in referral of patients in our environment. Aspiration of an FB can be associated with significant morbidity, and this must be borne in mind when managing these patients.

The ongoing debate about the development of appropriate tertiary services in the developing world remains unresolved. ${ }^{[1-5]}$ The trend to subspecialise that is so prominent in the developed world is gaining ground in SA. One of the criticisms of this trend is that the developing-world environment cannot sustain subspecialist care. However, there is little doubt that subspecialist-directed care that follows clinical algorithms can be delivered effectively. Given the varied nature and potential complications of FB ingestion or aspiration, such children should be managed in specialised centres.

\section{Conclusion}

Ingested or aspirated $\mathrm{FBs}$ are a common problem in our environment. Our outcomes are in keeping with those reported from other international and national centres. Our management algorithms are appropriate and are followed by our staff. We consider that our specialist-led paediatric surgery service has contributed greatly to these excellent outcomes, and this supports our drive to advocate for these cases to be managed in specialised centres.

\section{Acknowledgements. None.}

Author contributions. NFM: main author, data collection, literature review and draft write-up; HM: paediatric surgeon specialist, clinical and

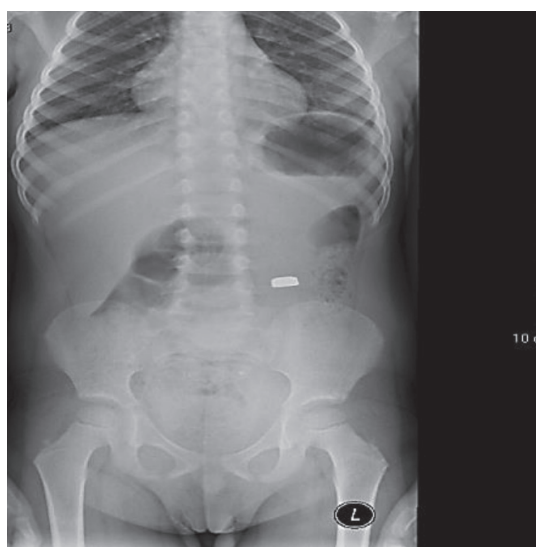

Fig. 7. A button battery in the small bowel. The patient underwent emergency laparotomy for removal to prevent bowel perforation.

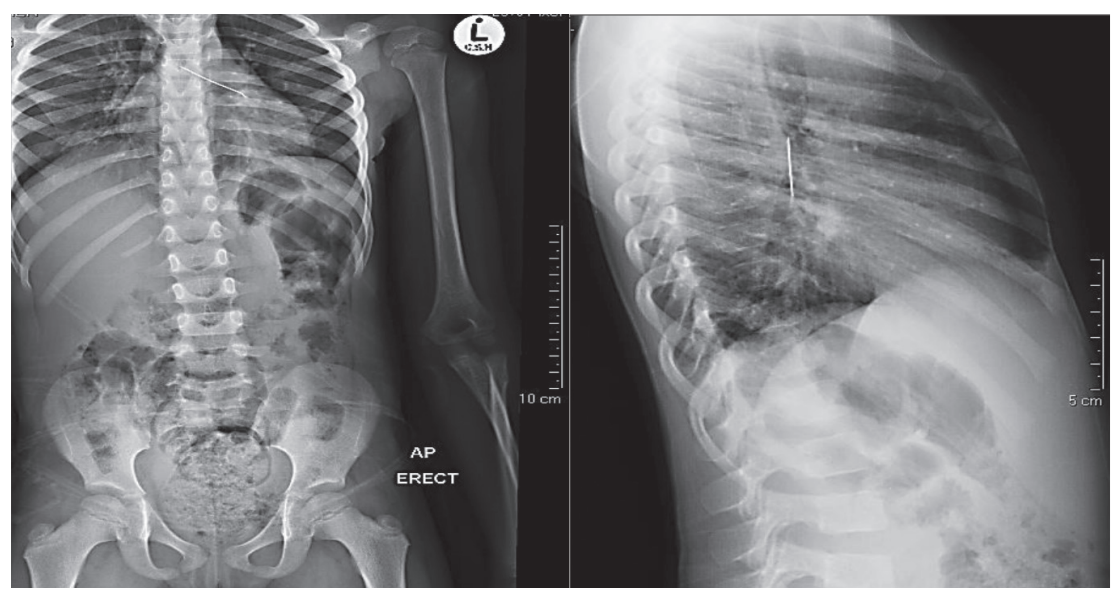

Fig. 8. Pin in the left main bronchus. The patient underwent thoracotomy for removal after failed removal via rigid bronchoscopy. 
expert advice; VG: data collection, proofreading and corrections; VYK: context and technical advisor; GLL: database manager and data collection; DLC: senior author, proof reading, corrections, final draft and overseeing the writing of the manuscript.

Funding. None.

\section{Conflicts of interest. None.}

1. Panieri E, Bass DH. The management of ingested foreign bodies in children - a review of 663 cases. Eur J Emerg Med 1995;2(2):83-87. https://doi.org/10.1097/00063110-199506000-00005

2. Sultan TA, van As AB. Review of tracheobronchial foreign body aspiration in the South African paediatric age group. J Thorac Dis 2016;8(12):3787-3796. https://doi.org/10.21037/jtd.2016.12.90

3. Van As AB, du Toit N, Wallis L, Stool D, Chen X, Rode H. The South African experience with ingestion injury in children. Int J Pediatr Otorhinolaryngol 2003;67(Suppl 1):S175-S178. https://doi. org/10.17140/j.ijporl.2003.12

4. Van As AB, Chen X, Millar AJ, Rode H. Ingestion and aspiration of foreign bodies in South African children. Afr Saf Promot 2002;1(2):43-47. https://doi.org/10.4314/asp.vli2.31551
ches
5. Van As AB, Yusof AM, Millar AJ; Susy Safe Working Group. Food foreign body injuries. Int J Pediatr Otorhinolaryngol 2012;76(Suppl 1):S20-S25. https://doi.org/10.1016/j.ijporl.2012.02.005

6. Naidoo RR, Reddi AA. Chronic retained foreign bodies in the esophagus. Ann Thorac Surg 2004;77(6):2218-2220. https://doi.org/10.1016/S0003-4975(03)01255-4

7. Jayachandra S, Eslick GD. A systematic review of paediatric foreign body ingestion: Presentation, . Jayachandra S, Eslick GD. A systematic review of paediatric foreign body ingestion: Presentation,
complications, and management. Int J Pediatr Otorhinolaryngol 2013;77(3):311-317. https://doi

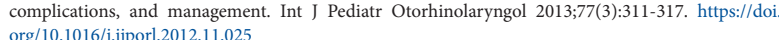
$\operatorname{org} / 10.1016 / j$.ijporl.2012.11.02 8. Kay M, Wyllie R. Pediatric foreign bodies and their management. Curr Gastroenterol Rep
2005;7(3):212-218. https://doi.org/10.1007/S11894-005-0037-6

9. Michaud L, Bellaïche M, Olives JP; Groupe Francophone d'Hépatologie, Gastroentérologie et Nutrition Pédiatriques (GFHGNP). Ingestion of foreign bodies in children: Recommendations o the French-Speaking Group of Pediatric Hepatology, Gastroenterology and Nutrition. Arch Pediatr 2009;16(1):54-61. https://doi.org/10.1016/j.arcped.2008.10.018

10. Shaariyah MM, Goh BS. Retrospective review of surgical management of foreign body ingestion. Med J Malays 2009;64(4):307-310.

Accepted 20 September 2017. 